Journal of Accident and Emergency Medicine 1994 11, 218-222

\title{
Temporal bone fractures in children: a review of 34
} cases

\author{
J.W.NICOL ${ }^{1}$ \& A.J.JOHNSTONE ${ }^{2}$ \\ ${ }^{1}$ Department of Paediatric Surgery, The Royal Hospital for Sick Children, Sciennes Road, Edinburgh and \\ ${ }^{2}$ Department of Paediatric Surgery, Western General Hospital, Crewe Road South, Edinburgh
}

\section{SUMMARY}

Head injuries are commonly seen in accident and emergency (A\&E) departments and within this group a small proportion will have a temporal bone fracture. Thirty-four such cases were identified from a 7-year period and their case notes were reviewed. The mechanisms of injury included:falls outdoors (15 cases), falls in the home (eight cases), road traffic accidents (RTAs; seven cases), missiles (three cases) and non-accidental injury (one case). In 20 cases the fracture involved more than one cranial bone, and the implications of this with regard to non-accidental injury are discussed. CT scans were carried out in 14 cases and 11 of these showed intracranial haematoma. The criteria for CT scan following head injury in general, and temporal bone fracture in particular are discussed. Outcome measures indicated that those injured as a result of RTAs had the poorest outcome, followed by those who fell outdoors and then those who fell in the home.

Key words: child, fracture, temporal

\section{INTRODUCTION}

Head injuries are commonly seen in A\&E departments, but within this group only a small number of patients will have a fracture of the temporal bone. Fractures of the temporal bone may present with haemotympanum, bleeding from the ear, cerebrospinal fluid (CSF) otorrhoea, perforation of the tympanic membrane, laceration of the ear canal or facial nerve paralysis. The diagnosis is often made on clinical grounds rather than radiographically, and the absence of an obvious temporal bone fracture on plain radiographs does not exclude the diagnosis. ${ }^{1}$

Temporal bone fractures may be complicated by intracranial haemorrhage, CSF leakage and infection, damage to the middle and inner ear and damage to the seventh and eighth cranial nerves. Classically, fractures of the temporal bone are described as being longitudinal or transverse, with respect to the axis of the petrous bone. ${ }^{2}$ Longitudinal fractures account for $80 \%$ of the total, and are usually associated with a temporary conductive hearing loss due to haemotympanum, and preservation of facial nerve function. In the remaining $20 \%$ where the fracture occurs transversely, there is more likely to be long-standing sensorineural hearing loss and facial nerve paralysis. The division into two distinct groups is rather artificial, the occurrence of a fracture along either of these planes being very unusual. More often, the fracture plane lies somewhere between the two extremes. It has been shown that true longitudinal fractures make up a much smaller proportion of the total than the classical description would suggest. ${ }^{3}$

Fractures of the temporal bone have been well described in the literature, but only one study has looked solely at temporal bone fractures as they affect children, and this was carried out in the USA. ${ }^{4}$ In general, children have a better outcome from a head injury of a given severity than adults, ${ }^{5}$ and therefore should be considered as a separate subpopulation. This paper describes temporal bone fractures as they affect a group of children in the UK.

\section{PATIENTS AND METHODS}

Up until 1991 two surgical paediatric units provided a service for a population of 92000 children less than 13 years of age. ${ }^{6}$ We identified all children under 13 years of age who had sustained a head injury resulting in death or admission to hospital in Edinburgh during the 7-year period between January 1983 and December 1989. Children with head injuries were admitted to hospital during this period according to the criteria accepted by the Working Party of the British Paediatric Association
Correspondence: GP Trainee, Hawick Health Centre, Hawick TD9 9DT, UK 
J.W. Nicol \& A.J. Johnstone
\& British Association of Paediatric Surgeons Joint Standing Commitee on Childhood Accidents. ${ }^{7}$ Data were obtained from Lothian Health Board records according to the diagnostic codes of the International Classification of Diseases (ninth revision) as: fracture of vault of skull, N800; fracture of base of skull, N801; other and unqualified skull fractures, N803, multiple fractures involving skull or face with other bones, N804; concussion, N850; cerebral laceration and contusion, N851; subarachnoid, subdural, and extradural haemorrhage following injury, N852; other and unspecified intracranial haemorrhage following injury, N853; intracranial injury of other and unspecified nature, N854 and other open wound of head, N873 subgroups $0,1,8$, 9. The case notes of all these patients were reviewed in order to confirm whether or not a fracture was associated with the head injury. The case notes of all children who sustained a fracture of the temporal bone were studied in detail.

In each case the diagnosis of temporal bone fracture was confirmed to have been made on the basis of clinical (sanguinous or CSF otorrhoea) or radiological findings (plain radiographs or CT scans), rather than on any less scientific basis. The following details of patients with temporal bone fractures were noted: age, sex, length of stay in hospital, presence and type of fracture, mechanism of injury, history of loss of consciousness, Glasgow Coma Scale (GCS) on admission and CT scan results (where performed). Outcome after treatment at the admission hospital was assessed using a modified Glasgow Outcome Scale (GOS, see Table 1).

\section{RESULTS}

In the 7-year study period 4487 children were admitted to hospital in Edinburgh after sustaining a

Table 1. A Modified Glasgow Outcome Scale (MGOS)

\begin{tabular}{lc}
\hline MGOS & Description \\
\hline 7 & Normal \\
6 & Deficit not impairing work or leisure \\
5 & Deficit impairing work or leisure \\
4 & Independent but unable to work or follow \\
3 & leisure pursuits \\
2 & Dependent but conscious \\
1 & Vegetative \\
\hline
\end{tabular}

head injury. Of these, 524 patients (12\%) had a skull fracture. Thirty-four patients had skull fractures involving the temporal bone, and accounted for less than $1 \%$ (34 out of 4487 ) of the group with head injuries, and $6 \%$ (34 out of 524) of the group with skull fractures. There were 21 boys and 13 girls. The age range was from 1 to 13 years, with a mean age of 6 years and a median age of 5 years. The length of stay in hospital ranged from 1 to 15 days, with a mean stay of 4 days, and a median stay of 2 days.

In 15 cases the fracture was the result of a fall outside the home. In nine of these cases the fracture occurred as a result of a fall at ground level whereas in six cases the children fell from a considerable height, for example, $3 \mathrm{~m}$ from a balcony, $3 \mathrm{~m}$ from a wall; $5 \mathrm{~m}$ through a roof; $13 \mathrm{~m}$ into a disused pool, $5 \mathrm{~m}$ from a window, and an unspecified fall of $4 \mathrm{~m}$. In eight cases the fall occurred in the home as follows: three children fell down a staircase; two fell from a kitchen work-surface; two fell from lounge furniture; and one fell off a bed striking her head on an electrical plug. Of the seven children who were involved in RTAs, six were pedestrians and one was a cyclist, all were struck by motor cars. In three cases the fracture was caused by a missile, including two swinging golf clubs and one falling roof-slate. One fracture was a non-accidental injury.

All the fractures resulting from falls within the home occurred in children aged 5 years or younger. Of the 15 children with a fracture resulting from falls outside the home, two-thirds were aged 5 years or older. Within the group of children involved in RTAs, four out of seven were aged 9 years or older. The child with the non-accidental injury was 2 months old.

In 14 patients the fracture involved only the temporal bone, while in the other 20 cases the fracture also involved other named regions of the skull. In the latter group it was most commonly a temporo-parietal fracture (nine cases) or a temporobasal fracture (five cases). The involvement of more than a single cranial bone, when associated with minor trauma, is a characteristic often found in abused children, ${ }^{8}$ so it is particularly important to recognize this pattern of fracture when physical abuse is suspected to be the cause. Among the 15 children who fell outdoors, a second cranial bone was fractured in four of those who fell from a considerable height and in five of those who fell at ground level. Of the nine children who fell in the home, four had this pattern of skull fracture, three having fallen down a staircase and one having 
Temporal bone

fractures in

children fallen from a kitchen work-surface. Fractures of this kind were also seen in five of the seven children who were involved in RTAs, in the child struck by a falling roof-slate, and in the single case of proven non-accidental injury.

From the histories given, 14 children were said to have lost consciousness, 14 were said not to have lost consiciousness and in six cases this aspect of the history was not recorded. The GCS was recorded in 31 cases, and in 22 of these the value was 15 . In five cases the value lay between three and eight, which represented a severe head injury. The other four cases had values ranging from nine to fourteen. Table 2 shows these data and groups the cases according to their mechanism of injury.

Fourteen patients had CT scans during their admission, and these are represented in Table 2 by an asterisk. The results of the CT scans are as follows: intracerebral haematoma (five cases), subarachnoid haematoma (three cases), subdural haematoma (two cases), extradural haematoma (one case) and contusion or swelling (three cases). Seven of the CT scans were performed in cases where the GCS on admission was below 15. Of these, four revealed intracranial haematoma, and three revealed contusion only. Five of the CT scans were carried out in cases where the GCS was 15 , the indication for the scan being as follows: the child who sustained the golf club injury, the child with non-accidental injury, and the child who fell onto an electrical plug all had depressed fractures; one child who fell down a staircase and a pedestrian who was struck by a motor car developed local neurological signs. CT scans were also carried out in two cases for whom there was no record of the GCS.

In terms of outcome 22 patients had a GOS of 7 . Of the 15 children who fell outdoors, four had a GOS below 7 and all these injuries involved falls from a considerable height. The child who fell $13 \mathrm{~m}$ into a disused pool was 8 years old; the CT scan showed a subarachnoid haematoma and the GOS was 6. A 3-year-old child fell $5 \mathrm{~m}$ from a window, had a CT scan showing an intracerebral haematoma, and a GOS of 4. A 9-year-old fell $5 \mathrm{~m}$ through a roof, had an intracerebral haematoma on CT scan and a GOS of 4 . One child fell $3 \mathrm{~m}$, had neither a CT scan nor a recorded GCS, and a GOS of 3.

Of the eight children who fell at home, two had GOS values of less than 7 . An 18-month-old child who fell down a staircase presented with a GCS of 7, had a CT scan showing diffuse swelling and a GOS of 4. A 5-year-old child who fell from a kitchen work-surface presented with a GCS of 15 and had a CT scan showing an intracerebral haematoma; after

\begin{tabular}{|c|c|c|c|}
\hline \multirow[b]{2}{*}{ Mechanism of injury } & \multicolumn{3}{|c|}{ Glasgow Coma Scale } \\
\hline & $\begin{array}{l}\text { Loss of } \\
\text { consciousness }\end{array}$ & $\begin{array}{c}\text { No loss of } \\
\text { consciousness }\end{array}$ & $\begin{array}{l}\text { No } \\
\text { record }\end{array}$ \\
\hline \multirow[t]{8}{*}{ Fall outdoors } & 15 & 15 & $?^{\star}$ (disused pool) \\
\hline & 15 & 15 & $?^{*}$ (window $15 \mathrm{~m}$ ) \\
\hline & 15 & 15 & \\
\hline & $14^{*}$ & 15 & \\
\hline & 9 & 15 & \\
\hline & $9^{*}$ (balcony $3 \mathrm{~m}$ ) & & \\
\hline & $6^{*}($ roof $5 \mathrm{~m})$ & & \\
\hline & $?$ (wall $3 \mathrm{~m}$ ) & & \\
\hline \multirow[t]{4}{*}{ Fall in the home } & 15 (stairs) & $15^{\star}$ (stairs) & $15^{\star}$ \\
\hline & $7^{\star}$ (stairs) & 15 & 15 (kitchen) \\
\hline & & 15 & \\
\hline & & 15 & \\
\hline Road traffic & $4^{*}$ & 15 (cyclist) & $15^{*}$ \\
\hline \multirow[t]{3}{*}{ accidents } & 3 & 15 & \\
\hline & & 15 & \\
\hline & & $4^{*}$ & \\
\hline Missile & $12^{*}$ (roof slate) & 15 & $15^{\star}$ (golf club) \\
\hline Non-accidental injury & $15^{\star}$ & & \\
\hline
\end{tabular}

Table 2. The Glasgow Coma Scales on admission for the different mechanisms of injury, grouped according to whether or not consciousness was lost ("Indicates patients who had CT scan) 
J.W. Nicol \& A.J. Johnstone management there remained a seventh nerve palsy so the GOS was 6.

In three out of seven children involved in RTAs the GOS was 7. A 9-year-old child with a GCS of 4 had a contusion on CT scan and the GOS was 6. Two children, aged 6 and 10 years, remained hemiplegic, so their GOS was 4; in one of these cases a CT scan was carried out and showed a subarachnoid haematoma. Another child, three years of age, presented with a GCS of three, and died.

Of those injured by missiles, the GOS was 7 in two cases, and three in the case of the 7-year-old boy who was struck by a falling roof-slate; his CT scan showed a contusion and he remained paraplegic. The baby who sustained a non-accidental injury had a subarachnoid haematoma on CT scan and $a$ GOS of 6 .

\section{DISCUSSION}

Many more children are discharged from A\&E departments following a head injury than are admitted, and within the group of children admitted to hospital in this study, less than $1 \%$ had a fracture involving the temporal bone. However, because of the possible serious complications, it is important that these fractures are recognized early, and that the appropriate management is instituted with regard to both the head injury itself and the otological and audiological outcome.

All the temporal fractures sustained in the home occurred in children who were 5 years of age or younger, whereas of those fractures sustained outside the home, two thirds occurred in children aged 5 years or older. In this respect temporal bone fractures in children are the same as most other childhood injuries and this probably reflects the new and wider range of hazards associated with the advent of school-age.

It is interesting to note that more often than not, the temporal bone fracture was associated with a fracture in another region of the skull (20 vs. 14). The significance of this pattern of fracture when associated with relatively minor trauma has already been mentioned. Non-accidental injury must be suspected, especially when there is an uncertain or varying history.

Excluding the six children whose notes did not include this aspect of the history, as many children did lose consciousness as did not. Table 2 shows that only one of those who did not lose consciousness had a GCS on admission of less than 15, and among those who did lose consciousness, only five had a GCS of 15. However, it would be dangerous to assume that remaining conscious after head injury is a reliable predictor of a high GCS on arrival at hospital. Considerable importance is often placed on this aspect of the history by a patient's relatives. To make this assumption, and be unaware of the possibility of a deteriorating conscious level after the initial injury, could lead to a delayed presentation to hospital.

CT scan is a useful method of diagnosing a temporal bone fracture, the sensitivity being as high as $90 \% .{ }^{9}$ However, as long as the conscious level is normal and there are no other local neurological signs, a patient with clinical evidence of a temporal bone fracture would only require a CT scan if there was a facial nerve palsy. This would demonstrate the site of the lesion prior to surgical decompression of the facial nerve. Thus, in the management of temporal bone fractures, a CT scan may be indicated occasionally by a seventh nerve lesion, but more commonly by signs arising from lesions caused by the head injury but not in the temporal bone.

Fourteen patients had CT scans carried out; in 11 there was a haematoma, and in three a contusion. This very high rate of positive CT scans raises the issue of whether more CT scans should have been carried out. In 1990 Teasdale et al. found that the main indicators of risk in paediatric head injuries are the same as in adults, namely a skull fracture and a sub-optimal conscious level. ${ }^{5}$ They recommended early CT scanning of more patients with head injuries. In particular, they urged that following a head injury, a patient with either a persisting alteration in conscious level or a skull fracture should have a CT scan. Between 1983 and 1989, the period of our study, there was a growing awareness of the benefits of computerized tomography as the facility became more widely available. Our high positive CT scan rate may reflect the conservative use of $C T$ scans in the early part of this period, and lends support to the arguement that they should play a far greater role in the management of head injuries.

In terms of outcome, 22 cases were normal and in 12 cases the GOS was less than seven. Of the different mechanisms of injury, RTAs led to the greatest proportion with poor outcomes (GOS $=6$, $4,4,1)$. Four out of 15 cases who fell outdoors had sub-optimal GOS values $(6,4,4,3)$, as did two of the eight children who fell in the home (GOS $=6$ and 4). Although our sample size is small, it appears that the poorest outcomes arise from RTAs, followed by falls outside, and then falls in the home. 
Temporal bone

fractures in

children

\section{REFERENCES}

1. Waldron J. \& Hurley S.E. (1988) Temporal bone fractures: a clinical diagnosis. Archives of Emergency Medicine 5(3), 146-150.

2. Proctor B., Gurdjian E.S. \& Webster J.E. (1956) The ear in head trauma. Laryngoscope 66, 16-59.

3. Ghorayeb B.Y. \& Yeakley J.W. (1992) Temporal bone fractures: longitudinal or oblique? The case for oblique temporal bone fractures. Laryngoscope 102(2), 129134.

4. McGuirt W.F. jr. \& Stool S.E. (1992) Temporal bone fractures in children: a review with emphasis on longterm sequelae. Clinical Pediatrics 31(1), 12-18.

5. Teasdale G.M., Murray G., Anderson E., Mendelow
A.D., MacMillan R., Jennett B. \& Brookes M. (1990) Risks of acute traumatic intracranial haematoma in children and adults: implications for managing head injuries. British Medical Journal 300, 363-367.

6. Registrar General (1991) General Register Office for Scotland. Ladywell House Edinburgh.

7. Lewis A.F. (ed.) (1983) The Management of Acute Head Injury. Harrogate Seminar Report 8. pp. 1-85. D.H.S.S., London.

8. Hobbs C.J. (1984) Skull fractures and the diagnosis of abuse. Archives of Disease in Childhood 59, 246-252.

9. Betz B.W. \& Wiener M.D. (1991) Air in the temporomandibular joint fossa: C.T. sign of temporal bone fracture. Radiology 180(2), 463-466. 